\title{
Variational Wigner-Kirkwood approach to relativistic mean field theory
}

\author{
M. Del Estal, M. Centelles, and X. Viñas \\ Departament d'Estructura i Constituents de la Matèria, Facultat de Física, Universitat de Barcelona, Diagonal 647, \\ E-08028 Barcelona, Spain \\ (Received 26 December 1996)
}

\begin{abstract}
The recently developed variational Wigner-Kirkwood approach is extended to the relativistic mean field theory for finite nuclei. A numerical application to the calculation of the surface energy coefficient in semiinfinite nuclear matter is presented. The new method is contrasted with the standard density functional theory and the fully quantal approach. [S0556-2813(97)01310-1]
\end{abstract}

PACS number(s): 21.60. $-\mathrm{n}, 2$ 1.10.Dr, 21.65. $+\mathrm{f}$

\section{INTRODUCTION}

Semiclassical methods are widely used to deal with properties of global character of different types of Fermi systems like atoms, nuclei, helium, or metallic clusters (see Refs. [1, 2] for comprehensive reviews). Concerning the nuclear ground-state energy, the simplest approach is the semiempirical mass formula based on the liquid drop model [3]. This approximation gives the smooth part of the energy and reproduces reasonably well the experimental values. The success of the mass formula is due to the fact that the fluctuating quantal correction to the nuclear energy (shell effects) is small as compared with the average part. This allows a perturbative treatment of the shell effects that is justified from a theoretical point of view by the Strutinsky energy theorem [4].

Actually, to obtain the correct semiclassical averaged energy one should solve the problem using the Strutinsky smoothing. However, this is in general more difficult to handle than the quantal problem itself if realistic nuclear potentials are used [5]. The search for alternative methods is therefore an interesting and yet open problem. One possibility is to use density functional theory (DFT) techniques, such as the Thomas-Fermi (TF) method and its extensions (ETF). These methods can be based on the Wigner-Kirkwood (WK) $\hbar$ expansion of the density matrix $[2,6]$. The WK theory provides an expansion of the particle and kinetic energy densities in gradients of the single-particle potential up to zeroth, second, or fourth order in $\hbar$. These $\hbar$ corrections come from the fact that in the Hamiltonian the momentum operator does not commute with the potential. In DFT the original WK expansion of the density $\rho$ is inverted to recast the kinetic energy density as a functional $\tau[\rho]$ of the density and its gradients. If the potential part of the interaction is also known as a functional of $\rho$, as happens with Skyrme forces, then minimization of the DFT energy leads to a variational equation for $\rho$ alone $[2,6]$.

Recently, the variational content of the WK expansion of the energy has been studied for a set of nonrelativistic fermions submitted to external (Woods-Saxon) or selfconsistent (Skyrme) one-body potentials [7-9]. It has been shown that the variational solution for the particle density that minimizes the semiclassical energy at each order in the $\hbar$ expansion is just the WK expansion of $\rho$ at the same order in $\hbar$. The method for solving this variational problem was presented in Ref. [7] and called variational WignerKirkwood (VWK) theory.

The VWK theory has been mainly applied to the calculation of the surface energy coefficient in semi-infinite nuclear matter (SINM). It has been found to reproduce the quantal value nicely [7-9]. From a comparison of VWK and DFT calculations $[2,9,10]$, one can see that the agreement with the quantal results is worse in DFT (even at order $\hbar^{4}$ ) than in VWK theory [7-9].

In recent years the investigation of nuclear systems by means of a relativistic approach has attracted a growing interest. Especially successful has been the phenomenological relativistic mean field theory (RMFT) [11]. The semiclassical approach to the relativistic theory has been recently worked out and $\hbar$ corrections to the earlier established relativistic TF model have been derived [12-14], both at the WK and at the relativistic DFT (RDFT) level. It is the purpose of this paper to formulate, to second order in $\hbar$, the relativistic variational Wigner-Kirkwood (RVWK) theory, i.e., the VWK approach to RMFT. We will do that based on the nonlinear $\sigma$ - $\omega$ model $[11,15]$. We do not attempt here to investigate the quality of the relativistic model. Rather, we want to compare the new semiclassical theory with the usual RDFT, adopting the quantal results as a standard, and try to find a good alternative to the Strutinsky averaging procedure. As an application of the theory we will calculate the surface energy coefficient in SINM.

\section{RELATIVISTIC VARIATIONAL WIGNER-KIRKWOOD THEORY}

Our starting point in setting the RVWK theory is the constrained energy $E_{c}$ of a finite nucleus:

$$
E_{c}=E-\lambda A=\int d \mathbf{r}\left[\mathcal{E}_{\mathrm{tot}}(\mathbf{r})-\lambda \rho(\mathbf{r})\right]
$$

where $\mathcal{E}_{\text {tot }}$ is the total energy density and the chemical potential $\lambda$ is the Lagrange multiplier that ensures the correct par- 
ticle number $A$. Assuming for simplicity the nucleus to be symmetric and uncharged, in the relativistic nonlinear $\sigma-\omega$ model $\mathcal{E}_{\text {tot }} \operatorname{reads}^{1}[11,15]$

$$
\begin{aligned}
\mathcal{E}_{\mathrm{tot}}= & \mathcal{E}+g_{v} V \rho-m \rho+\frac{1}{2}\left[(\boldsymbol{\nabla} \phi)^{2}+m_{s}^{2} \boldsymbol{\phi}^{2}\right] \\
& -\frac{1}{2}\left[(\boldsymbol{\nabla} V)^{2}+m_{v}^{2} V^{2}\right]+\frac{1}{3} b \phi^{3}+\frac{1}{4} c \phi^{4} .
\end{aligned}
$$

In this equation $V$ and $\phi$ are the vector and scalar fields, respectively, and the energy density $\mathcal{E}$ stands for $\tau+m \rho-g_{s} \phi \rho_{s}$, with $\tau$ the relativistic kinetic energy density and $\rho_{s}$ the scalar density. Quantally, we have $\rho=\Sigma_{\alpha} \varphi_{\alpha}^{\dagger} \varphi_{\alpha}$, $\rho_{s}=\Sigma_{\alpha} \varphi_{\alpha}^{\dagger} \beta \varphi_{\alpha}$, and $\tau=\Sigma_{\alpha} \varphi_{\alpha}^{\dagger}(-i \boldsymbol{\alpha} \cdot \boldsymbol{\nabla}+\beta m-m) \varphi_{\alpha}$ on a single-particle basis $\varphi_{\alpha}$. The free couplings and meson masses of the relativistic energy functional are usually fixed by adjusting them to reproduce nuclear matter properties or experimental data on finite nuclei within the Hartree approximation.

To second order in the WK expansion one has

$$
\rho=\rho_{0}+\rho_{2}, \quad \rho_{s}=\rho_{s, 0}+\rho_{s, 2}, \quad \mathcal{E}=\mathcal{E}_{0}+\mathcal{E}_{2} .
$$

The well-known relativistic TF expressions are

$$
\begin{gathered}
\rho_{0}=\frac{2 k_{F}^{3}}{3 \pi^{2}}, \\
\rho_{s, 0}=\frac{m^{*}}{\pi^{2}}\left[k_{F} \epsilon_{F}-m^{* 2} \ln \frac{k_{F}+\epsilon_{F}}{m^{*}}\right], \\
\mathcal{E}_{0}=\frac{1}{4 \pi^{2}}\left[k_{F} \epsilon_{F}^{3}+k_{F}^{3} \epsilon_{F}-m^{* 4} \ln \frac{k_{F}+\epsilon_{F}}{m^{*}}\right],
\end{gathered}
$$

with the definition of a local Fermi momentum

$$
k_{F}=\sqrt{\left(\lambda+m-g_{v} V\right)^{2}-m^{* 2}},
$$

a Dirac effective mass $m^{*}=m-g_{s} \phi$, and $\epsilon_{F}=\sqrt{k_{F}^{2}+m^{* 2}}$. The second-order WK corrections $\rho_{2}, \rho_{s, 2}$, and $\mathcal{E}_{2}$ contain squared gradients and Laplacians of the fields $V$ and $\phi$. Their expressions are considerably lengthier and will not be reproduced here as they can be found in Refs. [12-14]. On account of Eqs. (3) -(7), the WK constrained energy density $\mathcal{E}_{\text {tot }}-\lambda \rho$ becomes a functional of the vector and scalar fields only. This is in accordance with the fact that in the relativistic framework there exist two different densities, namely, the baryon and the scalar densities, in contrast to the nonrelativistic situation where the basic quantity is the ground-state density, related to the one-body potential through the Fermi momentum.

With Eq. (3), however, the constrained energy (1) does not represent yet a complete separation into $\hbar^{0}$ and $\hbar^{2}$ parts. The vector and scalar fields and the chemical potential also have to be split into zeroth- and second-order terms: $V=V_{0}+V_{2}, \phi=\phi_{0}+\phi_{2}$, and $\lambda=\lambda_{0}+\lambda_{2}$. For instance,

\footnotetext{
${ }^{1}$ Units $\hbar=c=1$ are used, except in the occasions where we write $\hbar$ to indicate the order of the semiclassical expansions.
}

$$
\begin{aligned}
\mathcal{E}= & \mathcal{E}_{0}(V, \phi, \lambda)+\mathcal{E}_{2}(V, \phi, \lambda)+\cdots \\
= & \mathcal{E}_{0}\left(V_{0}, \phi_{0}, \lambda_{0}\right)+\frac{\partial \mathcal{E}_{0}}{\partial V_{0}} V_{2}+\frac{\partial \mathcal{E}_{0}}{\partial \phi_{0}} \phi_{2}+\frac{\partial \mathcal{E}_{0}}{\partial \lambda_{0}} \lambda_{2} \\
& +\mathcal{E}_{2}\left(V_{0}, \phi_{0}, \lambda_{0}\right)+\mathcal{O}\left(\hbar^{4}\right)
\end{aligned}
$$

In what follows, the WK functionals $\rho_{0}, \rho_{2}, \mathcal{E}_{0}, \mathcal{E}_{2}$, etc., are to be evaluated at the $V_{0}, \phi_{0}$, and $\lambda_{0}$ values. Introducing $\mu=\lambda_{0}+m$, the separation of $E_{c}$ into pure $\mathrm{TF}\left(\hbar^{0}\right)$ and second-order $\left(\hbar^{2}\right)$ contributions will then read

$$
\begin{aligned}
E_{c}^{(0)}= & \int d \mathbf{r}\left\{\mathcal{E}_{0}+\left(g_{v} V_{0}-\mu\right) \rho_{0}+\frac{1}{2}\left[\left(\nabla \phi_{0}\right)^{2}+m_{s}^{2} \phi_{0}^{2}\right]\right. \\
& \left.-\frac{1}{2}\left[\left(\nabla V_{0}\right)^{2}+m_{v}^{2} V_{0}^{2}\right]+\frac{1}{3} b \phi_{0}^{3}+\frac{1}{4} c \phi_{0}^{4}\right\}, \\
E_{c}^{(2)}= & \int d \mathbf{r}\left\{\mathcal{E}_{2}+\frac{\partial \mathcal{E}_{0}}{\partial V_{0}} V_{2}+\frac{\partial \mathcal{E}_{0}}{\partial \phi_{0}} \phi_{2}+\frac{\partial \mathcal{E}_{0}}{\partial \lambda_{0}} \lambda_{2}\right. \\
& +\left(g_{v} V_{0}-\mu\right)\left(\rho_{2}+\frac{\partial \rho_{0}}{\partial V_{0}} V_{2}+\frac{\partial \rho_{0}}{\partial \phi_{0}} \phi_{2}+\frac{\partial \rho_{0}}{\partial \lambda_{0}} \lambda_{2}\right) \\
& +\left(g_{v} V_{2}-\lambda_{2}\right) \rho_{0}-\phi_{2}\left(\nabla^{2}-m_{s}^{2}\right) \phi_{0}+V_{2}\left(\nabla^{2}-m_{v}^{2}\right) V_{0} \\
& \left.+\left(b \phi_{0}^{2}+c \phi_{0}^{3}\right) \phi_{2}\right\} .
\end{aligned}
$$

Straightforward evaluation of the derivatives in $E_{c}^{(2)}$ simplifies it to

$$
\begin{aligned}
E_{c}^{(2)}= & \int d \mathbf{r}\left\{\mathcal{E}_{2}+\left(g_{v} V_{0}-\mu\right) \rho_{2}+\left(g_{v} V_{2}-\lambda_{2}\right) \rho_{0}-g_{s} \phi_{2} \rho_{s, 0}\right. \\
& -\phi_{2}\left(\nabla^{2}-m_{s}^{2}\right) \phi_{0}+V_{2}\left(\nabla^{2}-m_{v}^{2}\right) V_{0} \\
& \left.+\left(b \phi_{0}^{2}+c \phi_{0}^{3}\right) \phi_{2}\right\} .
\end{aligned}
$$

The gist of the derivation of the variational equations from the constrained energy $E_{c}=E_{c}^{(0)}+E_{c}^{(2)}+\mathcal{O}\left(\hbar^{4}\right)$ in the RVWK theory is the fact that the minimization must be performed for each order in $\hbar$ separately [7] (i.e., $\delta E_{c}^{(0)}=0$, $\left.\delta E_{c}^{(2)}=0\right)$. This point is carefully discussed in the Appendix. Defining $\mathcal{E}_{i}^{\text {eff }} \equiv \mathcal{E}_{i}+\left(g_{v} V_{0}-\mu\right) \rho_{i}$ with $i=0,2$ the following relationships hold:

$$
\frac{\delta \mathcal{E}_{i}^{\mathrm{eff}}}{\delta V_{0}}=g_{v} \rho_{i}, \quad \frac{\delta \mathcal{E}_{i}^{\mathrm{eff}}}{\delta \phi_{0}}=-g_{s} \rho_{s, i}
$$

With the aid of Eqs. (12), at lowest order one gets from Eq. (9) the usual Euler-Lagrange equations for the TF fields $V_{0}$ and $\phi_{0}[11,15]$ :

$$
\begin{gathered}
\left(\nabla^{2}-m_{v}^{2}\right) V_{0}+g_{v} \rho_{0}=0 \\
\left(\nabla^{2}-m_{s}^{2}\right) \phi_{0}+g_{s} \rho_{s, 0}-b \phi_{0}^{2}-c \phi_{0}^{3}=0 .
\end{gathered}
$$

The Fermi momentum at the TF level is easily found from Eq. (7): $k_{0}=\sqrt{\left(\mu-g_{v} V_{0}\right)^{2}-m_{0}^{* 2}}$. It should be noticed that the density is already normalized at the TF level [7] (see the Appendix for details): 


$$
A=\int d \mathbf{r} \rho_{0} .
$$

Consequently, the full $\hbar^{2}$ contribution to $A$ must vanish:

$$
\begin{gathered}
\int d \mathbf{r}\left[\rho_{2}+\frac{\partial \rho_{0}}{\partial V_{0}} V_{2}+\frac{\partial \rho_{0}}{\partial \phi_{0}} \phi_{2}+\frac{\partial \rho_{0}}{\partial \lambda_{0}} \lambda_{2}\right] \\
=\int d \mathbf{r}\left[\rho_{2}+\frac{\partial \rho_{0}}{\partial k_{0}} k_{2}\right]=0
\end{gathered}
$$

(see below for the correction $k_{2}$ to the Fermi momentum).

The second-order Euler-Lagrange equations are derived from Eq. (11) and, using Eqs. (12), turn out to be

$$
\begin{gathered}
\left(\nabla^{2}-m_{v}^{2}\right) V_{2}+g_{v} \rho_{2}-g_{s} \frac{\partial \rho_{s, 0}}{\partial V_{0}} \phi_{2}+\frac{\partial \rho_{0}}{\partial V_{0}}\left(g_{v} V_{2}-\lambda_{2}\right)=0 \\
\left(\nabla^{2}-m_{s}^{2}\right) \phi_{2}+g_{s} \rho_{s, 2}-\left(2 b \phi_{0}+3 c \phi_{0}^{2}-g_{s} \frac{\partial \rho_{s, 0}}{\partial \phi_{0}}\right) \phi_{2} \\
-\frac{\partial \rho_{0}}{\partial \phi_{0}}\left(g_{v} V_{2}-\lambda_{2}\right)=0 .
\end{gathered}
$$

In practice, the procedure to solve these variational equations for a finite nucleus with $A$ particles is the following. First, one solves the TF equations (13)-(15) in the usual manner [11] to find the fields $V_{0}$ and $\phi_{0}$ and the chemical potential $\lambda_{0}$. Inserting in Eqs. (17) and (18) the TF solutions, one gets two linear differential equations to compute the so-far unknown corrections $V_{2}$ and $\phi_{2}$. These quantities will depend on $\lambda_{2}$, which in turn has to be determined so that the normalization condition (16) is satisfied. Equations (16)-(18) will be iterated until consistency is reached. We will show below, however, that to calculate the correction of order $\hbar^{2}$ to the energy it is not necessary to obtain $V_{2}, \phi_{2}$, and $\lambda_{2}$; only the TF solutions $V_{0}, \phi_{0}$, and $\lambda_{0}$ are needed.

Once the solutions for the fields and the chemical potential are known, it is immediate to obtain the Fermi momentum to second order by direct expansion of Eq. (7):

$$
\begin{aligned}
k_{F}= & k_{0}+k_{2}=\sqrt{\left(\mu-g_{v} V_{0}\right)^{2}-m_{0}^{* 2}}+\frac{\epsilon_{0}}{k_{0}}\left(\lambda_{2}-g_{v} V_{2}\right) \\
& +g_{s} \frac{m_{0}^{*}}{k_{0}} \phi_{2} .
\end{aligned}
$$

In principle, due to divergences at the classical turning point, in RVWK theory the densities and potentials beyond the TF order must be considered as distributions. However, they are very efficient to compute expectation values by integrals over the space $[6,12,16]$.

It is worthwhile noting that the same variational equations (13), (14) and (17), (18) can be obtained if we start by directly varying Eq. (1) with respect to $V$ and $\phi$, now employing expressions (4) for $\rho$ and (6) for $\mathcal{E}$, and only afterwards do we perform in the new Euler-Lagrange equations the splitting of $V, \phi$, and $\lambda$ into their $\hbar^{0}$ and $\hbar^{2}$ parts. Thus, expansion and variation can be interchanged. For brevity, we prove this statement for the vector field equation only. The variation with respect to $V$ of Eq. (1) before expansion results in

$$
\left(\nabla^{2}-m_{v}^{2}\right) V+g_{v} \rho=0,
$$

with $\rho$ given by Eq. (4). Now one makes $V=V_{0}+V_{2}$ and $\rho=\rho_{0}+\left(\partial \rho_{0} / \partial V_{0}\right) V_{2}+\left(\partial \rho_{0} / \partial \phi_{0}\right) \phi_{2}+\left(\partial \rho_{0} / \partial \lambda_{0}\right) \lambda_{2}+\rho_{2}$. It is simple to see that

$$
\begin{gathered}
g_{v} \frac{\partial \rho_{0}}{\partial \phi_{0}}=g_{v} g_{s} \frac{2}{\pi^{2}} k_{0} m_{0}^{*}=-g_{s} \frac{\partial \rho_{s, 0}}{\partial V_{0}}, \\
g_{v} \frac{\partial \rho_{0}}{\partial \lambda_{0}}=g_{v} \frac{2}{\pi^{2}} k_{0} \epsilon_{0}=-\frac{\partial \rho_{0}}{\partial V_{0}} .
\end{gathered}
$$

Using these results and separating Eq. (20) into each order allows one to recover the previous variational equations (13) and (17).

On the other hand, one can follow an alternative route that consists in working with $k_{F}$ as if it were an independent variable of $V$ and $\phi$, i.e., without replacing it by Eq. (7). In this case, after expanding to order $\hbar^{2}\left(k_{F}=k_{0}+k_{2}\right)$, minimization with respect to $k_{0}\left(\delta E_{c}^{(0)} / \delta k_{0}=\delta E_{c}^{(2)} / \delta k_{0}=0\right)$ yields two variational equations which are just the zeroth- and second-order contributions to $k_{F}$ given by Eq. (19). Therefore, at each order in $\hbar$, the relation (7) between $k_{F}$ and the fields $V$ and $\phi$ is the variational Euler-Lagrange equation for $k_{F}$, a basic test of consistency. It also can be shown that when $k_{F}$ is kept as an independent variable, the variational equations for the fields reduce to Eqs. (13), (14) and (17), (18).

On the basis of Eqs. (13) and (14) it is easy to verify that several contributions to Eq. (11) cancel out so that, taking into account the condition (15), the correction of order $\hbar^{2}$ to the total energy $E=E_{c}+\lambda A$ finally is

$$
E^{(2)}=\int d \mathbf{r}\left[\mathcal{E}_{2}+\left(g_{v} V_{0}-\mu\right) \rho_{2}\right] .
$$

Since the quantities $\mathcal{E}_{2}$ and $\rho_{2}$ are to be evaluated using the $V_{0}$ and $\phi_{0}$ values, we arrive at the remarkable result that to calculate the energy to second order only the lowest-order TF equations (13) and (14) need to be solved. In this sense we have a perturbational approach, as the calculation of the energy to a given order in $\hbar^{2}$ requires knowledge of the solution only to the next lower order. A similar idea of including $\hbar^{2}$ corrections perturbatively, but in a rather more heuristic way, has been carried out in Ref. [17]. In particular, this calculation differs from the present method in that the chemical potential is not split into $\hbar^{0}$ and $\hbar^{2}$ parts.

Though we have derived the VWK equations for the relativistic energy functional (2), it is easy to realize that the method is more general and that it can be applied to more realistic functionals (e.g., with an isovector $\rho$ meson, other types of scalar couplings, or self-interactions of the vector field). To conclude this section, we would like to mention again that the semiclassical calculation provides only the average part of the quantal energy. However, a semiclassical method can be useful to replace some complicated full quantal calculations [6]. In such cases the remaining shell correc- 
tion should be added perturbatively to the semiclassical quantity, as indicated by the Strutinsky procedure or the expectation value method $[2,5,18]$.

\section{COMPARISON WITH RELATIVISTIC DENSITY FUNCTIONAL THEORY}

The normalization condition (15) brings us to the discussion of an important difference between the RVWK theory we have just introduced and the standard RDFT [12,19,20]. While in RVWK theory the result of the integral (16) vanishes, RDFT is more restrictive and reinforces this condition by imposing that the integrand vanishes locally. Then, in RDFT one has that, at each point,

$$
\widetilde{k_{2}}=-\frac{\pi^{2}}{2 \widetilde{k}_{0}^{2}} \rho_{2}
$$

We write $\widetilde{k_{0}}$ and $\widetilde{k_{2}}$ to distinguish them from $k_{0}$ and $k_{2}$ of the RVWK theory. Owing to the ansatz (24), in RDFT the functional $\rho_{0}\left(\widetilde{k_{0}}\right)$ equals the exact density $\rho$, i.e., $\rho=2 \widetilde{k}_{0}^{3} / 3 \pi^{2}$. Notice that at the TF level, RVWK theory and RDFT are equivalent.

Next we briefly recall the derivation of the RDFT variational equations. In RDFT the scalar and vector fields and the chemical potential are not explicitly split into $\hbar^{0}$ and $\hbar^{2}$ parts $[12,19,20]$; only $k_{F}$ is. Thus, expanding Eq. (2) into $\widetilde{k_{0}}$ and $\widetilde{k_{2}}$ and utilizing Eq. (24), the RDFT constrained energy to second order is

$$
\begin{aligned}
E_{c}^{\mathrm{RDFT}}= & \int d \mathbf{r}\left\{\mathcal{E}_{0}+\widetilde{\mathcal{E}}_{2}-\widetilde{\boldsymbol{\epsilon}}_{0}{\widetilde{\boldsymbol{\rho}_{2}}}+g_{v} \rho V-(\lambda+m) \rho\right. \\
& +\frac{1}{2}\left[(\boldsymbol{\nabla} \phi)^{2}+m_{s}^{2} \boldsymbol{\phi}^{2}\right]-\frac{1}{2}\left[(\boldsymbol{\nabla} V)^{2}+m_{v}^{2} V^{2}\right] \\
& \left.+\frac{1}{3} b \phi^{3}+\frac{1}{4} c \phi^{4}\right\},
\end{aligned}
$$

with $\widetilde{\epsilon}_{0}=\sqrt{\widetilde{k}_{0}^{2}+m^{* 2}}$. The notation $\widetilde{\mathcal{E}}_{2}$ and $\widetilde{\rho}_{2}$ means that in the WK expressions for $\mathcal{E}_{2}$ and $\rho_{2}$, the gradients of the field $V$ have been inverted in favor of the gradients of $\widetilde{k_{0}}$ and $\phi$ (or $\rho$ and $m^{*}$ ) as described in Refs. $[12,19,20]$, where the expression of the functional $\mathcal{E}_{2}^{\mathrm{RDFT}} \equiv \widetilde{\mathcal{E}}_{2}-\widetilde{\epsilon}_{0} \widetilde{\rho}_{2}$ can be found. From Eq. (25), the RDFT variational equation for $\widetilde{k_{0}}$ $\left(\delta E_{c}^{\mathrm{RDFT}} / \delta \widetilde{k_{0}}=0\right)$ becomes $[12,19,20]$

$$
\widetilde{\epsilon_{0}}+g_{v} V-\lambda-m-\frac{\pi^{2}}{2 \widetilde{k_{0}} \widetilde{\epsilon}_{0}} \widetilde{\rho_{2}}=0 .
$$

Actually, Eq. (26) corresponds again to the development of Eq. (7) for $k_{F}$ (equivalently, $\epsilon_{F}=\mu-g_{v} V$ ) into $\widetilde{k}_{0}$ and $\widetilde{k}_{2}$ : If we expand $\epsilon_{F}$ as $\widetilde{\epsilon}_{0}+\widetilde{\epsilon}_{2}$, the corrective term is just $\tilde{\epsilon}_{2}=-\pi^{2} \tilde{\rho}_{2} / 2 \widetilde{k_{0}} \tilde{\epsilon}_{0}$. The RDFT variational equations for the vector and scalar fields read $[12,19,20]$

$$
\begin{gathered}
\left(\nabla^{2}-m_{v}^{2}\right) V+g_{v} \rho=0, \\
\left(\nabla^{2}-m_{s}^{2}\right) \phi+g_{s} \rho_{s}^{\mathrm{RDFT}}-b \phi^{2}-c \phi^{3}=0,
\end{gathered}
$$

where

$$
\rho_{s}^{\mathrm{RDFT}}=\rho_{s, 0}+{\widetilde{\rho_{s, 2}}}-\frac{m^{*}}{\widetilde{\epsilon_{0}}} \widetilde{\rho_{2}}
$$

and ${\widetilde{\rho_{s, 2}}}_{\widetilde{\varepsilon}_{2}}$ indicates that the gradients of $V$ have been inverted as in $\widetilde{\mathcal{E}}_{2}$ and $\widetilde{\rho_{2}}$. Note that having varied the energy (25) as a whole, and not independently for each order in $\hbar$, the final RDFT solution of Eqs. (26)-(28) mixes different powers of $\hbar$. Nevertheless, the RDFT functionals are free from divergence problems at the classical turning point and generally provide a good description of the local density profile $[2,7,12]$.

\section{NUMERICAL APPLICATION}

To exemplify all the above on a concrete case, we shall present numerical calculations of the surface energy coefficient in SINM for several parameter sets of the relativistic interaction. The semi-infinite system corresponds to a onedimensional geometry where half the space is filled with nuclear matter at saturation and the other half is empty. The particle density then varies only along one axis, e.g., the $z$ axis, and develops a surface around $z=0$. Following Ref. [3], the surface energy coefficient $E_{s}$ in SINM is written as

$$
E_{s}=4 \pi r_{\infty}^{2} \int_{-\infty}^{\infty} d z\left[\mathcal{E}_{\mathrm{tot}}(z)-a_{v} \rho(z)\right],
$$

where $r_{\infty}$ and $a_{v}$ refer to the radius and energy per particle in saturated nuclear matter. In the self-consistent problem for SINM, $a_{v}$ equals the chemical potential $\lambda$, owing to the Hugenholtz-Van Hove theorem. Consequently, the surface energy is stationary with respect to variations of the density. Our previous formulation can be fully applied to the onedimensional semi-infinite geometry [compare Eqs. (1) and (30)] with the simplification that $\lambda$ is fixed and thus $\lambda_{2}=0$.

First, we will discuss SINM results for the linear $\sigma-\omega$ model $(b=c=0)$. In spite of its simplicity, the linear model allows one to investigate the incidence of the gradient corrections more easily. Second, we will consider the more general nonlinear $\sigma-\omega$ model. We are mainly interested in extracting the average part of the energy associated with $\sigma-\omega$ interactions whose parameters have been obtained from a mean field calculation. Within the framework of the RMFT, and taking the quantal Hartree calculations as a standard, the authors of Refs. [12, 18-21] studied extensively the quality of the relativistic TF and RDFT (to order $\hbar^{2}$ ) approximations, and how the results depend on the parametrization of the effective interaction. It was seen that one must analyze more than just one single parameter set to draw conclusions about the quality of the semiclassical approximations, for it much depends on some properties of the interaction. For our application to the surface of SINM, the mass of the scalar meson, $m_{s}$, and the effective mass at saturation, $m_{\infty}^{*} / m$, have the major influence. The discussion that follows is not significantly altered by changing the remaining saturation properties if they lie within ordinary values [19-21].

Table I collects the results for the surface energy coefficient $E_{s}$ calculated in the linear $\sigma-\omega$ model. Table II shows the corresponding surface thickness $t$ of the density profile 
TABLE I. Linear $\sigma-\omega$ model. Surface energy coefficient in the quantal Hartree approach, $E_{s}^{\mathrm{H}}$, and difference of the relativistic TF, DFT, and VKW calculations to $E_{s}^{\mathrm{H}}$. All quantities are in $\mathrm{MeV}$.

\begin{tabular}{ccccc}
\hline \hline$m_{s}$ & $E_{s}^{\mathrm{H}}$ & $E_{s}^{\mathrm{TF}}-E_{s}^{\mathrm{H}}$ & $E_{s}^{\mathrm{DFT}}-E_{s}^{\mathrm{H}}$ & $E_{s}^{\mathrm{VWK}}-E_{s}^{\mathrm{H}}$ \\
\hline 400 & 40.48 & 1.74 & -0.74 & 0.22 \\
450 & 33.85 & 2.06 & -1.00 & 0.29 \\
500 & 28.19 & 2.41 & -1.42 & 0.35 \\
550 & 23.05 & 2.96 & -2.12 & 0.57 \\
\hline \hline
\end{tabular}

(standard $90 \%$ to $10 \%$ falloff distance). The nuclear matter properties of the linear model are as follows [20]: volume energy $a_{v}=-15.75 \mathrm{MeV}$, density $\rho_{\infty}=0.193 \mathrm{fm}^{-3}$, incompressibility $K=546 \mathrm{MeV}$, and effective mass $m_{\infty}^{*} / m=0.56$. The values in Table I correspond to the discussed semiclassical approaches (TF, RVWK, and RDFT) and the fully quantal Hartree $(\mathrm{H})$ calculation. (See Ref. [22] for details on the quantal treatment of relativistic SINM.) In any WK calculation beyond the TF order one is faced with dealing with divergences at the turning point. The main difficulty is to treat them in such a way that the principal part of the energy can be extracted. In practice, we encountered only one divergent term in the WK functional $\mathcal{E}_{2}$. To get rid of the divergence we added and subtracted the analytical asymptotic integrand, so that after having isolated the infinity we rejected it. Other possibilities are the method of integrals in the complex plane [16] or low-temperature expansions [2].

The saturation properties of infinite nuclear matter are governed by the meson coupling-to-mass ratios $g_{s}^{2} / m_{s}^{2}$ and $g_{v}^{2} / m_{v}^{2}$ and by the nonlinear couplings $b$ and $c[11,15]$. On the contrary, the nuclear surface properties extracted from SINM depend on the meson coupling constants and masses separately. The mass of the vector meson, $m_{v}$, is given its physical value $(783 \mathrm{MeV})$. The mass of the scalar meson, $m_{s}$, should lie somewhere between 400 and $700 \mathrm{MeV}$, since the nonexperimental $\sigma$ particle is interpreted as simulating two-pion exchange contributions. For our purposes, it will be sufficient to look at the region $400 \mathrm{MeV} \leqslant m_{s} \leqslant 550 \mathrm{MeV}$. The scalar mass sets the range of the scalar interaction and, therefore, there is a strong correlation of $E_{s}$ and $t$ with $m_{s}$. A larger $m_{s}$ determines a shorter range of the attractive potential, leading to a steeper surface and to smaller $E_{s}$ and $t$, as seen from Tables I and II.

The TF surface energy coefficients in Table I overestimate the quantal ones from $\sim 4 \%$ for $m_{s}=400 \mathrm{MeV}$ to $\sim 13 \%$ for $m_{s}=550 \mathrm{MeV}$. When the $\hbar^{2}$ gradient corrections are taken into account, one finds that the surface energies calculated in the RVWK approach are larger than the $\mathrm{H}$ results, whereas the RDFT energies are smaller than in $\mathrm{H}$ calculations. In both cases $E_{s}$ is brought closer to the $\mathrm{H}$ value

TABLE II. Linear $\sigma$ - $\omega$ model. Surface thickness $t$ (in fm).

\begin{tabular}{llll}
\hline \hline$m_{s}$ & $t^{\mathrm{H}}$ & $t^{\mathrm{TF}}$ & $t^{\mathrm{DFT}}$ \\
\hline 400 & 2.98 & 3.22 & 2.86 \\
450 & 2.38 & 2.71 & 2.27 \\
500 & 1.94 & 2.27 & 1.72 \\
550 & 1.64 & 1.88 & 1.30 \\
\hline \hline
\end{tabular}

than in the TF calculation. In RVWK theory the deviations lie between $0.5 \% \quad\left(m_{s}=400 \mathrm{MeV}\right)$ and $2.5 \%$ $\left(m_{s}=550 \mathrm{MeV}\right)$, while in RDFT they range from $2 \%$ $\left(m_{s}=400 \mathrm{MeV}\right)$ to $9 \%\left(m_{s}=550 \mathrm{MeV}\right)$. From Table I one can check that the differences $E_{s}^{\mathrm{TF}}-E_{s}^{\mathrm{DFT}}$ and $E_{s}^{\mathrm{TF}}-E_{s}^{\mathrm{VWK}}$ show an upward tendency with the scalar mass $m_{s}$, and that $E_{s}^{\mathrm{TF}}-E_{s}^{\mathrm{DFT}}$ is systematically larger than $E_{s}^{\mathrm{TF}}-E_{s}^{\mathrm{VWK}}$. These trends can be qualitatively understood looking at the values of the surface thickness $t$ in Table II. The inhomogeneity corrections of RVWK theory and RDFT concentrate at the nuclear surface, where the gradients are more important. A flatter surface (small $m_{s}$, large $t$ ) results in smaller corrections. In RVWK theory one calculates the gradients with the TF density distributions that have a larger thickness than the RDFT profiles. Therefore, one expects the RVWK modifications to the TF energy to be smaller.

For relativistic harmonic oscillator scalar and vector fields, it has been numerically shown [12] that the energies calculated using the Strutinsky average and the WK approach to second order almost coincide, a well-known fact in the nonrelativistic frame [5]. It is thus reasonable to identify approximately the difference between $\mathrm{H}$ and RVWK calculations in the self-consistent problem with the quantal effects (in SINM, Friedel oscillations, and the fluctuating part of the spin-orbit force $[21,22]$ ). Even though the quantal surface energy coefficient is acceptably reproduced by RDFT in general, the difference with $\mathrm{H}$ calculations is larger than in RVWK theory and displays a stronger dependence on the particular value of $m_{s}$. Altogether RVWK theory appears as more reliable to estimate the quantal effects. This feature, also found in the nonrelativistic context [7], stems from the following reasons. First, RVWK theory properly sorts out the different orders in $\hbar$. Second, the restrictive local condition (24) for normalization within RDFT is replaced by the more logical global condition (16) in RVWK theory.

The surface energy is also strongly correlated with the value of the effective mass in nuclear matter $m_{\infty}^{*} / \mathrm{m}$ $[9,19,20]$. To analyze this fact we have considered the nonlinear parameter sets of Ref. [20]. They have $a_{v}=-15.75 \mathrm{MeV}, \rho_{\infty}=0.16 \mathrm{fm}^{-3}$, and $K=200 \mathrm{MeV}$, with $0.55 \leqslant m_{\infty}^{*} / m \leqslant 0.80$ and $400 \mathrm{MeV} \leqslant m_{s} \leqslant 550 \mathrm{MeV}$ which covers the range of commonly accepted values for $m_{\infty}^{*} / m$ and $m_{s}$. Figure 1 illustrates the dependence of the difference between the TF and $\mathrm{H}$ surface energy coefficients on $m_{\infty}^{*} / m$ and $m_{s}$. The discrepancies between the TF and $\mathrm{H}$ results exhibit a nearly linear behavior with $m_{\infty}^{*} / m$. For small values of the effective mass the TF surface energy coefficients are larger than the $\mathrm{H}$ ones. They practically agree with the $\mathrm{H}$ results for $m_{\infty}^{*} / m \simeq 0.65$, and become smaller than the $\mathrm{H}$ results for larger $m_{\infty}^{*} / m$. These trends have been found in a similar fashion for the total energies of finite nuclei in the $\sigma-\omega$ model [18] and in nonrelativistic calculations [9].

Figure 2 displays the difference in the surface energy coefficient between the semiclassical approaches to secondorder and $\mathrm{H}$ calculations. Again, for all the analyzed parameter sets, we observe that the RVWK energies are larger than the $\mathrm{H}$ ones while the RDFT energies are smaller. Also, the deviations to the $\mathrm{H}$ values are an increasing function of the scalar mass $m_{s}$. Their dependence on $m_{s}$ and on $m_{\infty}^{*} / m$ is weaker in RVWK theory than in RDFT (and, in both 


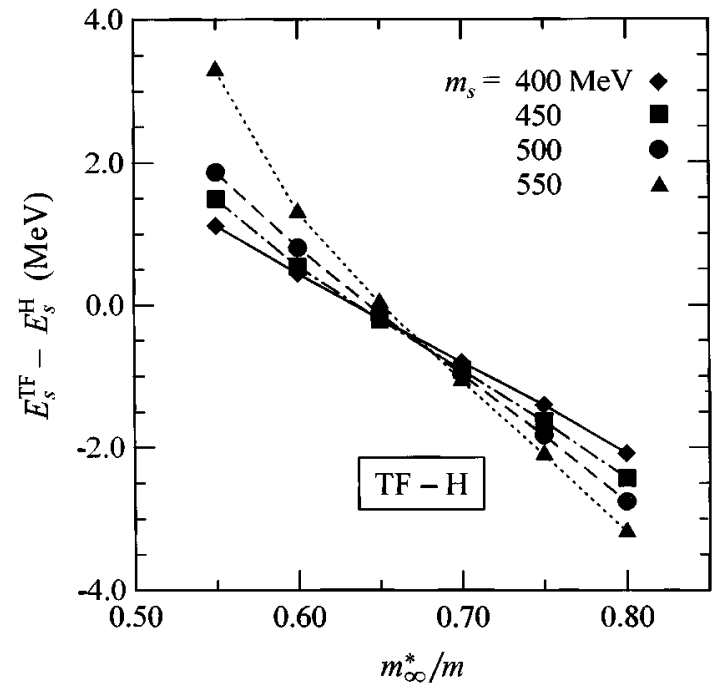

FIG. 1. Nonlinear $\sigma$ - $\omega$ model. Difference between the surface energy coefficients obtained in the relativistic TF and $\mathrm{H}$ approximations, as a function of the effective mass at saturation $m_{\infty}^{*} / m$ and the scalar mass $m_{s}$.

approaches, much weaker than in the TF method). Finally, one realizes that the semiclassical surface energies can show large discrepancies with the quantal value for some parameter sets (e.g., $m_{s}=550 \mathrm{MeV}$ and $m_{\infty}^{*} / m=0.55$ and 0.60 ). This is due to the fact that in such cases the thickness of the particle density and local effective mass is very small. Their gradients are therefore very large, causing the semiclassical expansions to break down.

\section{SUMMARY}

In this paper we have developed the relativistic variational Wigner-Kirkwood theory, extending first work in the nonrelativistic domain. This generalization is not trivial because of the presence of two different fields. The RVWK theory has

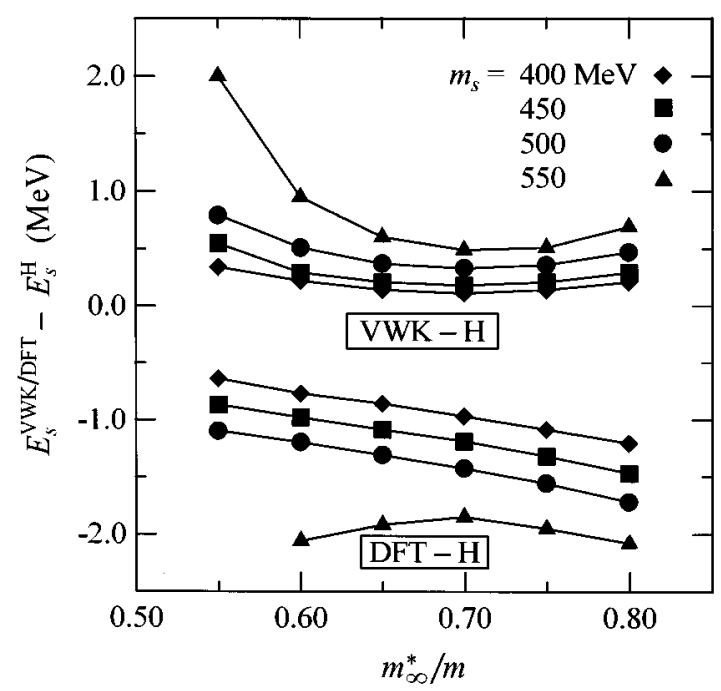

FIG. 2. Same as Fig. 1 for the difference of the second-order VWK and DFT calculations to the $\mathrm{H}$ results. A stable numerical solution could not be achieved in the DFT case when $m_{\infty}^{*} / m=0.55$ and $m_{s}=550 \mathrm{MeV}$, and hence this result is not shown. been based on a strict expansion of the energy in powers of $\hbar$, together with a global normalization to the particle number. Self-consistency enters at the TF level, whose solution is the input to calculate the higher-order corrections. In obtaining the variational equations we have shown that the steps of variation and expansion can be interchanged. We also have discussed the equivalence of working with the scalar and vector fields as the fundamental variables or with the Fermi momentum as an additional variable. The new theory has been compared with the RDFT.

Semi-infinite nuclear matter calculations in the relativistic problem have shown that the average part of the quantal surface energy is acceptably estimated in both the RVWK and RDFT approaches. However, for a quantitative estimate of the quantal effects, it has been seen that the RVWK theory is preferable. In addition, it must be considered an advantage of RVWK theory that its quality is less dependent on the properties of the effective interaction than in the TF and RDFT approximations.

\section{ACKNOWLEDGMENTS}

This work has been partially supported by the Catalan DGR Grant No. GRQ94-1022 and the Spanish DGICYT Grant No. PB95-1249. M.D.E. acknowledges financial support from the CIRIT (Catalonia). We are very grateful to Prof. P. Ring for providing a computer program for Hartree calculations on which we have based our version, and to Prof. P. Schuck and Prof. M. Durand for valuable discussions.

\section{APPENDIX}

Without loss of generality the variational problem addressed in Sec. II can be formulated as follows. Let $f[\psi]$ and $g[\psi]$ be two functionals of $\psi(\mathbf{r})$. Suppose they admit an expansion in powers of a small parameter (say, $\hbar$ ) and consider the problem of finding the function $\psi$ which extremizes

$$
\int d \mathbf{r} f[\psi]
$$

subject to the constraint

$$
\int d \mathbf{r} g[\psi]=A,
$$

up to a given order in the expansion (say, second order). For this purpose one constructs the auxiliary functional

$$
K[\psi, \lambda]=\int d \mathbf{r} f[\psi]-\lambda\left[\int d \mathbf{r} g[\psi]-A\right],
$$

where $\lambda$ is called a Lagrange multiplier. In obvious notation, expansion of $K[\psi, \lambda]$ to second order in the small parameter gives $K=K_{0}+K_{2}$ with

$$
K_{0}\left[\psi_{0}, \lambda_{0}\right]=\int d \mathbf{r}\left\{f_{0}\left[\psi_{0}\right]-\lambda_{0} g_{0}\left[\psi_{0}\right]\right\}+\lambda_{0} A,
$$




$$
\begin{aligned}
K_{2}\left[\psi_{0}, \psi_{2}, \lambda_{0}, \lambda_{2}\right]= & \int d \mathbf{r}\left\{f_{2}\left[\psi_{0}\right]-\lambda_{0} g_{2}\left[\psi_{0}\right]\right. \\
& \left.+\frac{\delta}{\delta \psi_{0}}\left(f_{0}-\lambda_{0} g_{0}\right) \psi_{2}-\lambda_{2} g_{0}\left[\psi_{0}\right]\right\} \\
& +\lambda_{2} A
\end{aligned}
$$

According to textbook theory of Lagrange multipliers, to locate the extrema of Eq. (A1) with the restriction (A2), one has to seek the critical points of the auxiliary functional $K$. In doing this, one treats $\psi_{0}$ and $\psi_{2}$ as independent variables because the constraint has already been taken into account through the Lagrange multipliers. Thus, we have

$$
\frac{\delta K}{\delta \psi_{0}}=\frac{\delta K}{\delta \psi_{2}}=0, \quad \frac{\partial K}{\partial \lambda_{0}}=\frac{\partial K}{\partial \lambda_{2}}=0 .
$$

The variation of $K=K_{0}+K_{2}$ with respect to $\psi_{0}$ produces

$$
\begin{gathered}
\frac{\delta}{\delta \psi_{0}}\left(f_{0}-\lambda_{0} g_{0}\right)+\frac{\delta}{\delta \psi_{0}}\left(f_{2}-\lambda_{0} g_{2}-\lambda_{2} g_{0}\right) \\
+\frac{\delta}{\delta \psi_{0}}\left[\frac{\delta}{\delta \psi_{0}}\left(f_{0}-\lambda_{0} g_{0}\right) \psi_{2}\right]=0,
\end{gathered}
$$

and from the variation with respect to $\psi_{2}$ one obtains

$$
\frac{\delta}{\delta \psi_{0}}\left(f_{0}-\lambda_{0} g_{0}\right)=0
$$

The derivatives on $\lambda_{0}$ and $\lambda_{2}$ yield, respectively,

$$
\begin{gathered}
\int d \mathbf{r}\left\{g_{0}\left[\psi_{0}\right]+g_{2}\left[\psi_{0}\right]+\frac{\delta g_{0}}{\delta \psi_{0}} \psi_{2}\right\}=A, \\
\int d \mathbf{r} g_{0}\left[\psi_{0}\right]=A .
\end{gathered}
$$

Equation (A8) is just

$$
\frac{\delta K_{0}}{\delta \psi_{0}}=0
$$

Inserting Eq. (A8) into Eq. (A7) shows that Eq. (A7) is equivalent to

$$
\frac{\delta K_{2}}{\delta \psi_{0}}=0 .
$$

Equation (A10) tells us that the lowest order already fulfills the restriction condition (A2), and in view of Eq. (A9) the total second-order contribution to $A$ vanishes:

$$
\int d \mathbf{r}\left\{g_{2}\left[\psi_{0}\right]+\frac{\delta g_{0}}{\delta \psi_{0}} \psi_{2}\right\}=0 .
$$

Therefore, one can paraphrase the variational problem under consideration as follows. Minimization must be performed for each order in the expansion parameter separately, Eqs. (A11) and (A12), and the constraint must be satisfied by the zeroth-order solution. Once $\psi_{0}$ and $\lambda_{0}$ are known by solving Eqs. (A10) and (A11), they are inserted into Eqs. (A12) and (A13) to calculate the second-order corrections $\psi_{2}$ and $\lambda_{2}$.

Expanding Eq. (A1),

$$
\int d \mathbf{r} f[\psi]=\int d \mathbf{r}\left\{f_{0}\left[\psi_{0}\right]+f_{2}\left[\psi_{0}\right]+\frac{\delta f_{0}}{\delta \psi_{0}} \psi_{2}\right\} .
$$

Using Eqs. (A8) and (A13),

$$
\int d \mathbf{r} \frac{\delta f_{0}}{\delta \psi_{0}} \psi_{2}=-\lambda_{0} \int d \mathbf{r} g_{2}\left[\psi_{0}\right]
$$

And, finally,

$$
\int d \mathbf{r} f[\psi]=\int d \mathbf{r}\left\{f_{0}\left[\psi_{0}\right]+f_{2}\left[\psi_{0}\right]-\lambda_{0} g_{2}\left[\psi_{0}\right]\right\} .
$$

This result shows that the extremum of Eq. (A1) can be computed to second order from the knowledge of $\psi_{0}$ and $\lambda_{0}$ only. We then observe that the whole procedure is consistent with the spirit of perturbation theory, since the lowest-order solution serves as the input to calculate the higher-order corrections. It furthermore guarantees that different powers of the expansion parameter do not mix at each order of the expansion.
[1] Theory of the Inhomogeneous Electron Gas, edited by S. D. Lundquist and N. H. March (Plenum, New York, 1983); R. O. Jones and O. Gunnarsson, Rev. Mod. Phys. 61, 689 (1989); R. M. Dreizler and E. K. U. Gross, Density Functional Theory (Springer, Berlin, 1990); Density Functional Theory, edited by E. K. U. Gross and R. M. Dreizler, Vol. 337 of NATO Advanced Study Institute Series B: Physics (Plenum, New York, 1995).

[2] B. Grammaticos and A. Voros, Ann. Phys. (N.Y.) 123, 359 (1979); Ann. Phys. (N.Y.) 129, 153 (1980); M. Brack, C. Guet, and H.-B. Håkansson, Phys. Rep. 123, 275 (1985); M. Centelles, M. Pi, X. Viñas, F. Garcias, and M. Barranco, Nucl. Phys. A510, 397 (1990).

[3] W. D. Myers and W. J. Swiatecki, Ann. Phys. (N.Y.) 55, 395
(1969); Ann. Phys. (N.Y.) 84, 186 (1974).

[4] V. M. Strutinsky, Nucl. Phys. A95, 420 (1967); Nucl. Phys. A122, 1 (1968).

[5] M. Brack and H. C. Pauli, Nucl. Phys. A207, 401 (1973); B. K. Jennings, ibid. A207, 538 (1973); B. K. Jennings, R. K. Bhaduri, and M. Brack, ibid. A253, 29 (1975).

[6] P. Ring and P. Schuck, The Nuclear Many-Body Problem (Springer-Verlag, New York, 1980), Chap. 13.

[7] P. Schuck and X. Viñas, Phys. Lett. B 302, 1 (1993).

[8] M. Durand, P. Schuck, and X. Viñas, Z. Phys. A 346, 87 (1993).

[9] X. Viñas, M. Centelles, M. Durand, and P. Schuck, in Proceedings of the International Conference on Many-Body Physics, Coimbra, 1993, edited by C. Fiolhais et al. (World Scientific, Singapore, 1994), p. 383. 
[10] J. Treiner and H. Krivine, Ann. Phys. (N.Y.) 170, 406 (1986); W. Stocker, J. Bartel, J. R. Nix, and A. J. Sierk, Nucl. Phys. A489, 252 (1988).

[11] B. D. Serot and J. D. Walecka, Adv. Nucl. Phys. 16, 1 (1986); B. D. Serot, Rep. Prog. Phys. 55, 1855 (1992).

[12] M. Centelles, X. Viñas, M. Barranco, and P. Schuck, Nucl. Phys. A519, 73c (1990); Ann. Phys. (N.Y.) 221, 165 (1993).

[13] D. Von-Eiff, S. Haddad, and M. K. Weigel, Phys. Rev. C 46, 230 (1992).

[14] C. Speicher, R. M. Dreizler, and E. Engel, Ann. Phys. (N.Y.) 213, 312 (1992).

[15] J. Boguta and A. R. Bodmer, Nucl. Phys. A292, 413 (1977).
[16] H. Krivine, M. Casas, and J. Martorell, Ann. Phys. (N.Y.) 200, 304 (1990).

[17] D. Von-Eiff and M. K. Weigel, Phys. Rev. C 46, 1797 (1992).

[18] M. Centelles, X. Viñas, M. Barranco, S. Marcos, and R. J. Lombard, Nucl. Phys. A537, 486 (1992).

[19] C. Speicher, E. Engel, and R. M. Dreizler, Nucl. Phys. A562, 569 (1993).

[20] M. Centelles and X. Viñas, Nucl. Phys. A563, 173 (1993).

[21] D. Von-Eiff, W. Stocker, and M. K. Weigel, Phys. Rev. C 50, 1436 (1994).

[22] D. Hofer and W. Stocker, Nucl. Phys. A492, 637 (1989). 